\title{
Exact solution for motion of an Oldroyd-B fluid over an infinite flat plate that applies an oscillating shear stress to the fluid
}

\author{
Nazish Shahid ${ }^{1 *}$, Mehwish Rana ${ }^{1}$ and Imran Siddique ${ }^{2}$
}

\author{
* Correspondence: \\ nash_shhd@hotmail.com \\ 'Abdus Salam School of \\ Mathematical Sciences, GC \\ University, 68-B New Muslim Town, \\ Lahore, Pakistan \\ Full list of author information is \\ available at the end of the article
}

\begin{abstract}
The unsteady motion of an Oldroyd-B fluid over an infinite flat plate is studied by means of the Laplace and Fourier transforms. After time $t=0$, the plate applies cosine/sine oscillating shear stress to the fluid. The solutions that have been obtained are presented as a sum of steady-state and transient solutions and can be easily reduced to the similar solutions corresponding to Newtonian or Maxwell fluids. They describe the motion of the fluid some time after its initiation. After that time when the transients disappear, the motion is described by the steady-state solutions that are periodic in time and independent of the initial conditions. Finally, the required time to reach the steady-state is established by graphical illustrations. It is lower for cosine oscillations in comparison with sine oscillations of the shear, decreases with respect to $\omega$ and $\lambda$ and increases with regard to $\lambda_{r}$.

Mathematical Subject Classification (2010): 76A05; 76A10.

PACS: 47.50.-d; 47.85.-g.
\end{abstract}

Keywords: Oldroyd-B fluid, oscillating shear stress, exact solutions

\section{Introduction}

The laminar flow of a great number of fluids such as polymeric liquids, food products, paints and so forth cannot be adequately described by means of the classical linearly viscous Newtonian model. The interest into motion problems of such fluids, also called non-Newtonian fluids, has considerably grown due to their multiple applications. Among the many models that have been used to describe the behavior of non-Newtonian fluids, the rate type models have received much attention. The first systematic thermodynamic study of such models is that of Rajagopal and Srinivasa [1], within which models for a variety of rate type viscoelastic fluids can be obtained. They showed that the Oldroyd-B fluid is one which stores energy like a linearized elastic solid, its dissipation however being due to two dissipative mechanisms that implies that they arise from a mixture of two viscous fluids. The first exact solutions corresponding to some motions of Oldroyd-B fluids seem to be those of Tanner [2] and Waters and King [3].

Over the past few decades, the unsteady flows of viscoelastic fluids caused by the oscillations of the boundary are of considerable interest. Rajagopal [4] found steadystate solutions for some oscillating motions of second grade fluids and Erdogan [5]

(c) 2012 Shahid et al; licensee Springer. This is an Open Access article distributed under the terms of the Creative Commons Attribution License (http://creativecommons.org/licenses/by/2.0), which permits unrestricted use, distribution, and reproduction in any medium, provided the original work is properly cited. 
provided two starting solutions for the motion of a viscous fluid due to cosine and sine oscillations of a flat plate. Flows of the fluids due to oscillating boundary for different constitutive models can be found into [6-20]. However, all these articles deal with motion problems in which the velocity is given on the boundary. To the best of our knowledge, the first exact solutions for motions of non-Newtonian fluids due to an infinite plate that applies a shear stress to the fluid seem to be those of Waters and King [21] and Bandelli et al. [22]. Recently [23], interesting solutions have been obtained for the motion of Newtonian fluids induced by an infinite plate that applies oscillating shear stresses to the fluid. This is very important as in some problems, what is specified is the force applied on the boundary. The "no slip" boundary condition may not be necessarily applicable to flows of polymeric fluids that can slip or slide on the boundary. Thus, the shear stress boundary condition is particularly meaningful and a closed-form expression for the starting solutions corresponding to the motion induced by a flat plate that applies an oscillating shear to an Oldroyd-B fluid has not been given before.

Consequently, the aim of this article is to determine starting solutions for the unsteady motion of an incompressible Oldroyd-B fluid due to an infinite plate that applies an oscillating shear to the fluid. Such exact solutions, which are not common in the literature, provide an important check for numerical methods that are used to study flows of such fluids in a complex domain. They are presented as a sum of steady-state and transient solutions and satisfy both the governing equations and all imposed initial and boundary conditions. Furthermore, the similar solutions for Maxwell and Newtonian fluids can easily be obtained as limiting cases of general solutions. Finally, the influence of the material parameters on the fluid motion and the required time to reach the steady-state are determined by graphical illustrations. This time is lower for the cosine oscillations in comparison with the sine oscillations of the shear, decreases with respect to the relaxation time $\lambda$ and the frequency $\omega$ of the shear and increases with respect to the retardation time $\lambda_{r}$.

\section{Governing equations}

An incompressible Oldroyd-B fluid is characterized by the following constitutive equations $[9,14,17]$

$$
\mathbf{T}=-p \mathbf{I}+\mathbf{S}, \quad \mathbf{S}+\lambda\left(\dot{\mathbf{S}}-\mathbf{L S}-\mathbf{S L}^{T}\right)=\mu\left[\mathbf{A}+\lambda_{r}\left(\dot{\mathbf{A}}-\mathbf{L A}-\mathbf{A L}^{T}\right)\right]
$$

where $\mathbf{T}$ is the Cauchy stress tensor, $-p \mathbf{I}$ denotes the indeterminate spherical stress, $\mathbf{S}$ is the extra-stress tensor, $\mathbf{L}$ is the velocity gradient, $\mathbf{A}=\mathbf{L}+\mathbf{L}^{T}$ is the first Rivlin-Ericksen tensor, $\mu$ is the dynamic viscosity, $\lambda$ and $\lambda_{r}$ are the relaxation and retardation times, the superscript $T$ indicates the transpose operation and the superposed dot denotes the material time derivative. In the following analysis, we will consider a unidirectional flow whose velocity field is given by

$$
\mathbf{V}=\mathbf{V}(\gamma, t)=u(y, t) \mathbf{i},
$$

where $\mathbf{i}$ denotes the unit vector along the $x$-direction of the Cartesian coordinate system $x, y$, and $z$. For such a flow, the constraint of incompressibility is automatically satisfied. We also assume that the extra-stress tensor $\mathbf{S}$, as well as the velocity $\mathbf{V}$, depends only on $y$ and $t$. In the absence of a pressure gradient in the flow direction 
and neglecting body forces, the governing equation is given by [14]

$$
\frac{\partial u(y, t)}{\partial t}+\lambda \frac{\partial^{2} u(y, t)}{\partial t^{2}}=v\left(1+\lambda_{r} \frac{\partial}{\partial t}\right) \frac{\partial^{2} u(y, t)}{\partial y^{2}} ; y, t>0,
$$

where $v=\mu / \rho$ is the kinematic viscosity and $\rho$ is the constant density of the fluid. The non-trivial shear stress $\tau(y, t)=S_{x y}(y, t)$ satisfies the partial differential equation [[24], Equation (4)]

$$
\left(1+\lambda \frac{\partial}{\partial t}\right) \tau(y, t)=\mu\left(1+\lambda_{r} \frac{\partial}{\partial t}\right) \frac{\partial u(\gamma, t)}{\partial y} ; \gamma, t>0 .
$$

\section{Statement of the problem}

Let us consider an incompressible Oldroyd-B fluid at rest, over an infinite plate. After time $t=0$ the plate applies an oscillating shear to the fluid $(f \sin \omega t$ or $f \cos \omega t$, where $f$ and $\omega$ are constants). Owing to the shear, the fluid is gradually moved. Its velocity has the form of Equation (2), the governing equation is given by Equation (3) and the appropriate initial and boundary conditions are

$$
\begin{aligned}
& u(y, 0)=0, \frac{\partial u(y, 0)}{\partial t}=0, \tau(y, 0)=0, y>0 \\
& \left.\left(1+\lambda \frac{\partial}{\partial t}\right) \tau(y, t)\right|_{y=0}=\left.\mu\left(1+\lambda_{r} \frac{\partial}{\partial t}\right) \frac{\partial u(y, t)}{\partial y}\right|_{y=0}=f \sin \omega t \operatorname{orf} \cos \omega t t>0 .
\end{aligned}
$$

Moreover, the natural condition

$$
u(y, t) \rightarrow 0 \text { as } y \rightarrow \infty
$$

also has to be satisfied.

\section{Exact solutions}

In the following, let us denote by $u_{s}(y, t), \tau_{s}(y, t)$ and $u_{c}(y, t), \tau_{c}(y, t)$, the solutions corresponding to the two problems and by

$$
V(\gamma, t)=u_{c}(y, t)+i u_{s}(\gamma, t), T(y, t)=\tau_{c}(\gamma, t)+i \tau_{s}(\gamma, t)
$$

the complex velocity and the complex tension, respectively. In view of the above equations, the functions $V(y, t)$ and $T(y, t)$ have to be solutions of the next initial and boundary values problems

$$
\begin{aligned}
& \frac{\partial V(y, t)}{\partial t}+\lambda \frac{\partial^{2} V(y, t)}{\partial t^{2}}=v\left(1+\lambda_{r} \frac{\partial}{\partial t}\right) \frac{\partial^{2} V(y, t)}{\partial y^{2}} y, t>0, \\
& \left(1+\lambda \frac{\partial}{\partial t}\right) T(y, t)=\mu\left(1+\lambda_{r} \frac{\partial}{\partial t}\right) \frac{\partial V(y, t)}{\partial y} y, t>0 \\
& V(y, 0)=0, \frac{\partial V(y, 0)}{\partial t}=0, T(y, 0)=0 y>0
\end{aligned}
$$




$$
\begin{aligned}
& \left.\left(1+\lambda \frac{\partial}{\partial t}\right) T(y, t)\right|_{y=0}=\left.\mu\left(1+\lambda_{r} \frac{\partial}{\partial t}\right) \frac{\partial V(y, t)}{\partial y}\right|_{y=0}=f e^{i \omega t} t>0, \\
& V(y, t) \rightarrow 0 \text { as } y \rightarrow \infty t>0 .
\end{aligned}
$$

\subsection{Calculation of the velocity field}

In order to determine the solution of initial-boundary values problem (9), (11) $)_{1,2},(12)_{2}$ and (13), we first take the Laplace transform [25] of Equation (9) and obtain

$$
q \bar{V}(y, q)+\lambda q^{2} \bar{V}(y, q)=v\left(1+\lambda_{r} q\right) \frac{\partial^{2} \bar{V}(y, q)}{\partial y^{2}}
$$

where the Laplace transform $\bar{V}(y, q)$ of function $V(y, t)$ has to satisfy the conditions

$$
\begin{aligned}
& \left.\frac{\partial \bar{V}(y, q)}{\partial y}\right|_{y=0}=\frac{f}{\mu(q-i \omega)\left(1+\lambda_{r} q\right)}, \\
& \bar{V}(y, q) \rightarrow 0 \text { as } y \rightarrow \infty .
\end{aligned}
$$

Multiplying Equation (14) $\sqrt{\frac{2}{\pi}} \cos (y \xi)$, integrating the result with respect to $y$ from 0 to infinity and using Equations (15) and (16), we obtain

$$
\bar{V}_{c}(\xi, q)=-\sqrt{\frac{2}{\pi}} \frac{f}{\rho} \frac{1}{(q-i \omega)\left[\lambda q^{2}+\left(1+\lambda_{r} \nu \xi^{2}\right) q+\nu \xi^{2}\right]^{\prime}}
$$

where

$$
\bar{V}_{c}(\xi, q)=\sqrt{\frac{2}{\pi}} \int_{0}^{\infty} \bar{V}(y, q) \cos (\gamma \xi) d y,
$$

denotes the Fourier cosine transform [26] of function $\bar{V}(y, q)$. Equation (17) can be written as

$$
\bar{V}_{c}(\xi, q)=\bar{V}_{c 1}(\xi, q)+\bar{V}_{c 2}(\xi, q)
$$

where

$$
\begin{aligned}
& \bar{V}_{c 1}(\xi, q)=-\sqrt{\frac{2}{\pi}} \frac{f}{\rho} \frac{\left(\nu \xi^{2}-\lambda \omega^{2}\right)-i \omega\left(1+\lambda_{r} \nu \xi^{2}\right)}{\left(\nu \xi^{2}-\lambda \omega^{2}\right)^{2}+\omega^{2}\left(1+\lambda_{r} \nu \xi^{2}\right)^{2}} \frac{1}{q-i \omega} \\
& \bar{V}_{c 2}(\xi, q)=\sqrt{\frac{2}{\pi}} \frac{f}{\rho} \frac{\left(\nu \xi^{2}-\lambda \omega^{2}\right)-i \omega\left(1+\lambda_{r} \nu \xi^{2}\right)}{\left(\nu \xi^{2}-\lambda \omega^{2}\right)^{2}+\omega^{2}\left(1+\lambda_{r} \nu \xi^{2}\right)^{2}} \frac{\lambda q+\left[i \lambda \omega+\left(1+\lambda_{r} \nu \xi^{2}\right)\right]}{\lambda q^{2}+\left(1+\lambda_{r} \nu \xi^{2}\right) q+\nu \xi^{2}}
\end{aligned}
$$

Applying the inverse Laplace transform and then the inverse Fourier cosine transform to Equation (20), we get the following expression

$$
V_{1}(\gamma, t)=-\frac{2}{\pi} \frac{f e^{i \omega t}}{\rho \nu^{2} \alpha} \int_{0}^{\infty} \frac{\left(\nu \xi^{2}-\lambda \omega^{2}\right)-i \omega\left(1+\lambda_{r} \nu \xi^{2}\right)}{\left(\xi^{2}-\beta^{2}\right)^{2}+\gamma^{2}} \cos (\gamma \xi) d \xi,
$$


where

$$
\alpha=1+\lambda_{r}^{2} \omega^{2}, \beta^{2}=\frac{\omega^{2}\left(\lambda-\lambda_{r}\right)}{v\left(1+\lambda_{r}^{2} \omega^{2}\right)}, \gamma=\frac{\omega\left(1+\lambda \lambda_{r} \omega^{2}\right)}{v\left(1+\lambda_{r}^{2} \omega^{2}\right)} .
$$

Using $\left(A_{1}\right)$ and $\left(A_{2}\right)$ from Appendix 1, we obtain the following simplified expression

$$
V_{1}(y, t)=\frac{f}{\mu} \sqrt{\frac{v}{\omega}} \frac{e^{-\gamma B}}{\sqrt[4]{\left(1+\lambda_{r}^{2} \omega^{2}\right)\left(1+\lambda^{2} \omega^{2}\right)}}\left[\cos \left(\omega t-\gamma A+\varphi+\frac{\pi}{2}\right)+i \sin \left(\omega t-\gamma A+\varphi+\frac{\pi}{2}\right)\right]
$$

where

$$
\begin{gathered}
2 A^{2}=\sqrt{\beta^{4}+\gamma^{2}}+\beta^{2}, 2 B^{2}=\sqrt{\beta^{4}+\gamma^{2}}-\beta^{2} \text { and } \\
\tan \varphi=\frac{\sqrt{1+\lambda^{2} \omega^{2}}-\lambda \omega \sqrt{1+\lambda_{r}^{2} \omega^{2}}}{\sqrt{1+\lambda_{r}^{2} \omega^{2}}+\lambda_{r} \omega \sqrt{1+\lambda^{2} \omega^{2}}} .
\end{gathered}
$$

Now, for Equation (21), we introduce the function

$$
F(\xi, q)=\frac{\lambda q+\left[i \lambda \omega+\left(1+\lambda_{r} \nu \xi^{2}\right)\right]}{\lambda q^{2}+\left(1+\lambda_{r} \nu \xi^{2}\right) q+\nu \xi^{2}}
$$

which can be written in the following equivalent form

$$
F(\xi, q)=\frac{q+\frac{b(\xi)}{2 \lambda}}{\left(q+\frac{b(\xi)}{2 \lambda}\right)^{2}-\left(\frac{c(\xi)}{2 \lambda}\right)^{2}}+\frac{b(\xi)+i 2 \lambda \omega}{c(\xi)} \frac{\frac{c(\xi)}{2 \lambda}}{\left(q+\frac{b(\xi)}{2 \lambda}\right)^{2}-\left(\frac{c(\xi)}{2 \lambda}\right)^{2}}
$$

where

$$
b(\xi)=1+\lambda_{r} \nu \xi^{2}, c(\xi)=\sqrt{\left(1+\lambda_{r} \nu \xi^{2}\right)^{2}-4 \nu \lambda \xi^{2}} .
$$

Applying the inverse Laplace transform and then the inverse Fourier transform to Equation (21) and using Equation (25), we obtain the following expression

$$
\begin{gathered}
V_{2}(y, t)=\frac{2}{\pi} \frac{f}{v \mu \alpha} \int_{0}^{\infty} \frac{\cos (\gamma \xi)}{\left(\xi^{2}-\beta^{2}\right)^{2}+\gamma^{2}}\left[\left(\nu \xi^{2}-\lambda \omega^{2}\right) c h\left(\frac{c(\xi) t}{2 \lambda}\right)+\frac{\left(\nu \xi^{2}+\lambda \omega^{2}\right) b(\xi)}{c(\xi)} s h\left(\frac{c(\xi) t}{2 \lambda}\right)\right] e^{-\frac{b(\xi) t}{2 \lambda}} d \xi \\
-i \frac{2}{\pi} \frac{f \omega}{v \mu \alpha} \int_{0}^{\infty} \frac{\cos (\gamma \xi)}{\left(\xi^{2}-\beta^{2}\right)^{2}+\gamma^{2}}\left[b(\xi) c h\left(\frac{c(\xi) t}{2 \lambda}\right)+\frac{b^{2}(\xi)-2 \lambda\left(\nu \xi^{2}-\lambda \omega^{2}\right)}{c(\xi)} \operatorname{sh}\left(\frac{c(\xi) t}{2 \lambda}\right)\right] e^{-\frac{b(\xi) t}{2 \lambda}} d \xi .
\end{gathered}
$$

Finally, the velocity corresponding to the cosine oscillations of the shear is given by

$$
\begin{gathered}
u_{c}(\gamma, t)=\frac{f}{\mu} \sqrt{\frac{\nu}{\omega}} \frac{e^{-\gamma B}}{\sqrt[4]{\left(1+\lambda_{r}^{2} \omega^{2}\right)\left(1+\lambda^{2} \omega^{2}\right)}} \cos \left(\omega t-\gamma A+\varphi+\frac{\pi}{2}\right)+\frac{2}{\pi} \frac{f}{\nu \mu \alpha} \\
\times \int_{0}^{\infty} \frac{\cos (y \xi)}{\left(\xi^{2}-\beta^{2}\right)^{2}+\gamma^{2}}\left[\left(\nu \xi^{2}-\lambda \omega^{2}\right) c h\left(\frac{c(\xi) t}{2 \lambda}\right)+\frac{\left(\nu \xi^{2}+\lambda \omega^{2}\right) b(\xi)}{c(\xi)} \operatorname{sh}\left(\frac{c \xi) t}{2 \lambda}\right)\right] e^{-\frac{b(\xi) t}{2 \lambda}} d \xi,
\end{gathered}
$$

while that corresponding to sine oscillations has the form

$$
\begin{gathered}
u_{s}(y, t)=\frac{f}{\mu} \sqrt{\frac{\nu}{\omega}} \frac{e^{-\gamma B}}{\sqrt[4]{\left(1+\lambda_{r}^{2} \omega^{2}\right)\left(1+\lambda^{2} \omega^{2}\right)}} \sin \left(\omega t-\gamma A+\varphi+\frac{\pi}{2}\right)-\frac{2}{\pi} \frac{f \omega}{\nu \mu \alpha} \\
\times \int_{0}^{\infty} \frac{\cos (\gamma \xi)}{\left(\xi^{2}-\beta^{2}\right)^{2}+\gamma^{2}}\left[b(\xi) \operatorname{ch}\left(\frac{c(\xi) t}{2 \lambda}\right)+\frac{b^{2}(\xi)-2 \lambda\left(\nu \xi^{2}-\lambda \omega^{2}\right)}{c(\xi)} \operatorname{sh}\left(\frac{c(\xi) t}{2 \lambda}\right)\right] e^{-\frac{b(\xi) t}{2 \lambda}} d \xi .
\end{gathered}
$$


The starting solutions (27) and (28) corresponding to cosine and sine oscillations of the shear, are presented as a sum between the steady-state and transient solutions. They describe the motion of the fluid some time after its initiation. After that time, in which the transients disappear, the starting solutions tend to the steady-state solutions

$$
\begin{gathered}
u_{c s}(\gamma, t)=\frac{f}{\mu} \sqrt{\frac{\nu}{\omega}} \frac{e^{-\gamma B}}{\sqrt[4]{\left(1+\lambda_{r}^{2} \omega^{2}\right)\left(1+\lambda^{2} \omega^{2}\right)}} \cos \left(\omega t-\gamma A+\varphi+\frac{\pi}{2}\right) \\
=\frac{f}{\mu} \sqrt{\frac{\nu}{\omega}} \frac{e^{-\gamma B}}{\sqrt[4]{\left(1+\lambda_{r}^{2} \omega^{2}\right)\left(1+\lambda^{2} \omega^{2}\right)}} \sin (\omega t-\gamma A+\varphi+\pi),
\end{gathered}
$$

respectively,

$$
u_{s s}(\gamma, t)=\frac{f}{\mu} \sqrt{\frac{\nu}{\omega}} \frac{e^{-\gamma B}}{\sqrt[4]{\left(1+\lambda_{r}^{2} \omega^{2}\right)\left(1+\lambda^{2} \omega^{2}\right)}} \sin \left(\omega t-\gamma A+\varphi+\frac{\pi}{2}\right) .
$$

which are periodic in time and independent of the initial conditions. However, they satisfy the governing equation and boundary conditions. As expected, they differ with a phase shift.

\subsection{Calculation of shear stress}

In order to obtain the corresponding shear stresses, we apply the Laplace transform to Equation (10) and the inverse Fourier cosine transform to Equation (17). Combining the results, we get the following expression for the Laplace transform of the complex tension $T(y, t)$ :

$$
\bar{T}(\gamma, q)=\frac{2}{\pi} \frac{f \mu}{\rho} \int_{0}^{\infty} \xi \sin (\gamma \xi) \frac{1}{1+\lambda q} \frac{1}{q-i \omega} \frac{\lambda_{r} q+1}{\lambda q^{2}+\left(1+\lambda_{r} \nu \xi^{2}\right) q+\nu \xi^{2}} d \xi .
$$

Equation (31) can be written as

$$
\bar{T}(y, q)=\bar{T}_{1}(\gamma, q)+\bar{T}_{2}(\gamma, q)+\bar{T}_{3}(\gamma, q),
$$

where

$$
\begin{aligned}
& \bar{T}_{1}(y, q)=\frac{-2 f}{\pi\left(1+\lambda^{2} \omega^{2}\right)} \frac{1-i \lambda \omega}{q+\frac{1}{\lambda}} \int_{0}^{\infty} \frac{\sin (\gamma \xi)}{\xi} d \xi \\
& \bar{T}_{2}(\gamma, q)=\frac{2 f}{\pi\left(1+\lambda^{2} \omega^{2}\right)} \frac{1}{q-i \omega} \int_{0}^{\infty} \xi \sin (\gamma \xi)\left[\frac{\xi^{2}-\beta^{2}-\lambda \omega \gamma}{\left(\xi^{2}-\beta^{2}\right)^{2}+\gamma^{2}}-i \frac{\lambda \omega \xi^{2}-\lambda \omega \beta^{2}+\gamma}{\left(\xi^{2}-\beta^{2}\right)^{2}+\gamma^{2}}\right] d \xi \\
& \bar{T}_{3}(\gamma, q)=\frac{2}{\pi} \frac{f \mu}{\rho} \int_{0}^{\infty} \xi \sin (\gamma \xi) \frac{M(\xi) q+N(\xi)}{\lambda q^{2}+\left(1+\lambda r \nu \xi^{2}\right) q+\nu \xi^{2}} d \xi
\end{aligned}
$$

with

$$
\begin{aligned}
& M(\xi)=\frac{\lambda}{\nu\left(1+\lambda^{2} \omega^{2}\right)}\left\{\frac{\xi^{2}\left(\lambda \omega \gamma-\beta^{2}\right)+\left(\gamma^{2}+\beta^{4}\right)+i\left[\xi^{2}\left(\gamma+\lambda \omega \beta^{2}\right)-\lambda \omega\left(\gamma^{2}+\beta^{4}\right)\right]}{\xi^{2}\left(\left(\xi^{2}-\beta^{2}\right)^{2}+\gamma^{2}\right)}\right\}, \\
& N(\xi)=\frac{1}{\omega\left(1+\lambda^{2} \omega^{2}\right)}\left\{\frac{-\xi^{2}\left(\gamma+\lambda \omega \beta^{2}\right)+\lambda \omega\left(\gamma^{2}+\beta^{4}\right)+i\left[\xi^{2}\left(\lambda \omega \gamma-\beta^{2}\right)+\left(\gamma^{2}+\beta^{4}\right)\right]}{\left.\left(\xi^{2}-\beta^{2}\right)^{2}+\gamma^{2}\right)}\right\} .
\end{aligned}
$$


Applying the inverse Laplace transform to the Equation (33) and using $\left(A_{3}\right)$ from Appendix 1, we get

$$
T_{1}(\gamma, t)=\left(\frac{-f}{1+\lambda^{2} \omega^{2}}+i \frac{f \lambda \omega}{1+\lambda^{2} \omega^{2}}\right) e^{\frac{-t}{\lambda}} .
$$

Similarly, using $\left(A_{4}\right)$ and $\left(A_{5}\right)$ from Appendix 1, from Equation (34), we obtain the following suitable form for $T_{2}(y, t)$

$$
T_{2}(y, t)=\frac{f e^{-\gamma B}}{1+\lambda^{2} \omega^{2}}[\cos (\omega t-\gamma A)+\lambda \omega \sin (\omega t-\gamma A)+i(\sin (\omega t-\gamma A)-\lambda \omega \cos (\omega t-\gamma A))] .
$$

A straightforward calculation leads to the following simplified form

$$
T_{2}(\gamma, t)=\frac{f e^{-\gamma B}}{\sqrt{1+\lambda^{2} \omega^{2}}}[\cos (\omega t-\gamma A-\psi)+i \sin (\omega t-\gamma A-\psi)]
$$

where $\tan \psi=\lambda \omega$.

Now, we consider the function

$$
G(\xi, q)=\frac{M(\xi) q+N(\xi)}{\lambda q^{2}+\left(1+\lambda_{r} \nu \xi^{2}\right) q+\nu \xi^{2}}
$$

which can be written in the following equivalent form:

$$
G(\xi, q)=M(\xi) \frac{q+\frac{b(\xi)}{2 \lambda}}{\lambda\left[\left(q+\frac{b(\xi)}{2 \lambda}\right)^{2}-\left(\frac{b(\xi)}{2 \lambda}\right)^{2}\right]}+\left[\frac{-b(\xi) M(\xi)}{\lambda c(\xi)}+\frac{2 N(\xi)}{c(\xi)}\right] \frac{\frac{c(\xi)}{2 \lambda}}{\left[\left(q+\frac{b(\xi)}{2 \lambda}\right)^{2}-\left(\frac{c(\xi)}{2 \lambda}\right)^{2}\right]} .
$$

Applying the inverse Laplace transform to Equation (35) and using Equation (40), we obtain

$$
\begin{gathered}
T_{3}(\gamma, t)=\frac{2 f}{\pi\left(1+\lambda^{2} \omega^{2}\right)} \int_{0}^{\infty} \frac{\sin (\gamma \xi)}{\xi\left[\left(\xi^{2}-\beta^{2}\right)^{2}+(\gamma)^{2}\right]}\left[\left(\left(\xi^{2}\left(\lambda \omega \gamma-\beta^{2}\right)+\left(\gamma^{2}+\beta^{4}\right)\right) c h\left(\frac{c(\xi) t}{2 \lambda}\right)-\frac{p(\xi)}{\omega c(\xi)} \operatorname{sh}\left(\frac{c \xi \xi) t}{2 \lambda}\right)\right)\right. \\
\left.\left.+i\left(\xi^{2}\left(\gamma+\lambda \omega \beta^{2}\right)-\lambda \omega\left(\gamma^{2}+\beta^{4}\right)\right] c h\left(\frac{c(\xi) t}{2 \lambda}\right)+\frac{r(\xi)}{\omega c(\xi)} s h\left(\frac{c(\xi) t}{2 \lambda}\right)\right)\right] e^{-\frac{b(\xi) t}{2 \lambda}} d \xi
\end{gathered}
$$

where

$$
\begin{gathered}
p(\xi)=\nu \xi^{4}\left[\lambda_{r} \omega\left(\lambda \omega \gamma-\beta^{2}\right)+2\left(\gamma+\lambda \omega \beta^{2}\right)\right]+\omega\left(\gamma^{2}+\beta^{4}\right)+\omega \xi^{2}\left[\left(\lambda \omega \gamma-\beta^{2}\right)-\nu\left(\gamma^{2}+\beta^{4}\right)\left(2 \lambda-\lambda_{r}\right)\right], \\
r(\xi)=\nu \xi^{4}\left[2\left(\lambda \omega \gamma-\beta^{2}\right)-\lambda_{r} \omega\left(\gamma+\lambda \omega \beta^{2}\right)\right]+\xi^{2}\left[\nu\left(\gamma^{2}+\beta^{4}\right)\left(2+\lambda \lambda_{r} \omega^{2}\right)-\omega\left(\gamma+\lambda \omega \beta^{2}\right)\right]+\lambda \omega^{2}\left(\gamma^{2}+\beta^{4}\right) .
\end{gathered}
$$

Using Equations (32), (36), (38), and (41), the shear stress corresponding to cosine oscillations of the shear can be written in the form

$$
\begin{aligned}
\tau_{c}(y, t)= & \frac{-f}{1+\lambda^{2} \omega^{2}} e^{\frac{-t}{\lambda}}+\frac{f e^{-\gamma \beta}}{\sqrt{1+\lambda^{2} \omega^{2}}} \cos (\omega t-\gamma A-\psi)+\frac{2 f}{\pi\left(1+\lambda^{2} \omega^{2}\right)} \int_{0}^{\infty} \frac{\sin (\gamma \xi)}{\xi\left[\left(\xi^{2}-\beta^{2}\right)^{2}+(\gamma)^{2}\right]} \\
& \times\left[\left(\xi^{2}\left(\lambda \omega \gamma-\beta^{2}\right)+\left(\gamma^{2}+\beta^{4}\right)\right) \operatorname{ch}\left(\frac{c(\xi) t}{2 \lambda}\right)-\frac{p(\xi)}{\omega c(\xi)} \operatorname{sh}\left(\frac{c(\xi) t}{2 \lambda}\right)\right] e^{\frac{-b(\xi) t}{2 \lambda} d \xi .}
\end{aligned}
$$

Also, the shear stress corresponding to sine oscillations is given by

$$
\begin{aligned}
\tau_{s}(y, t)= & \frac{f \lambda \omega}{1+\lambda^{2} \omega^{2}} e^{\frac{-t}{\lambda}}+\frac{f e^{-\gamma B}}{\sqrt{1+\lambda^{2} \omega^{2}}} \sin (\omega t-\gamma A-\psi)+\frac{2 f}{\pi\left(1+\lambda^{2} \omega^{2}\right)} \int_{0}^{\infty} \frac{\sin (\gamma \xi)}{\xi\left[\left(\xi^{2}-\beta^{2}\right)^{2}+(\gamma)^{2}\right]} \\
& \times\left[\left[\xi^{2}\left(\gamma+\lambda \omega \beta^{2}\right)-\lambda \omega\left(\gamma^{2}+\beta^{4}\right)\right] \operatorname{ch}\left(\frac{c(\xi) t}{2 \lambda}\right)+\frac{r(\xi)}{\omega c(\xi)} \operatorname{sh}\left(\frac{c(\xi) t}{2 \lambda}\right)\right] e^{\frac{-b(\xi) t}{2 \lambda}} d \xi .
\end{aligned}
$$


Of course, the shear stresses given by Equations (42) and (43) are also presented as a sum of steady-state and transient solutions. The steady-state solutions

$$
\tau_{c s}(y, t)=\frac{f e^{-\gamma B}}{\sqrt{1+\lambda^{2} \omega^{2}}} \cos (\omega t-\gamma A-\psi) \text { and } \tau_{s s}(y, t)=\frac{f e^{-\gamma B}}{\sqrt{1+\lambda^{2} \omega^{2}}} \sin (\omega t-\gamma A-\psi),
$$

also differ by a phase shift. The property seems to be not true for the transient solutions. This is the reason that we separately gave the starting solutions for both cosine and sine oscillations of the shear stress on the boundary.

\section{Particular cases}

\section{$5.1 \lambda_{r}=0$ (Maxwell fluid)}

Making $\lambda_{r} \rightarrow 0$ into Equations (27) and (28), we obtain the velocity fields

$$
\begin{gathered}
u_{c M}(y, t)=\frac{f}{\mu} \sqrt{\frac{\nu}{\omega}} \frac{e^{-\gamma B}}{\sqrt[4]{\left(1+\lambda^{2} \omega^{2}\right)}} \cos \left(\omega t-\gamma A+\varphi+\frac{\pi}{2}\right)+\frac{2 f}{\pi \mu \nu} \int_{0}^{\infty} \frac{\cos (\gamma \xi)}{\left(\xi^{2}-\frac{\lambda \omega^{2}}{\nu}\right)^{2}+\left(\frac{\omega}{v}\right)^{2}} \\
\times\left[\left(\nu \xi^{2}-\lambda \omega^{2}\right) \operatorname{ch}\left(\frac{\sqrt{1-4 \lambda \nu \xi^{2}}}{2 \lambda} t\right)+\frac{\nu \xi^{2}+\lambda \omega^{2}}{\sqrt{1-4 \lambda \nu \xi^{2}}} s h\left(\frac{\sqrt{1-4 \lambda \nu \xi^{2}}}{2 \lambda} t\right)\right] e^{-\frac{t}{2 \lambda}} d \xi, \\
u_{S M}(\gamma, t)=\frac{f}{\mu} \sqrt{\frac{\nu}{\omega}} \frac{e^{-\gamma B}}{\sqrt[4]{\left(1+\lambda^{2} \omega^{2}\right)}} \sin \left(\omega t-\gamma A+\varphi+\frac{\pi}{2}\right)-\frac{2}{\pi} \frac{f \omega}{\mu \nu} \int_{0}^{\infty} \frac{\cos (\gamma \xi)}{\left(\xi^{2}-\frac{\lambda \omega^{2}}{\nu}\right)^{2}+\left(\frac{\omega}{\nu}\right)^{2}} \\
\times\left[\operatorname{ch}\left(\frac{\sqrt{1-4 \lambda \nu \xi^{2}}}{2 \lambda} t\right)+\frac{1-2 \lambda\left(\nu \xi^{2}-\lambda \omega^{2}\right)}{\sqrt{1-4 \lambda \nu \xi^{2}}} \operatorname{sh}\left(\frac{\sqrt{1-4 \lambda \nu \xi^{2}}}{2 \lambda} t\right)\right] e^{-\frac{t}{2 \lambda} d \xi,}
\end{gathered}
$$

corresponding to a Maxwell fluid performing the same motion. Similarly, from Equations (42) and (43), we obtain the corresponding shear stresses

$$
\begin{aligned}
\tau_{c M}(y, t) & =-\frac{f}{1+\lambda^{2} \omega^{2}} e^{-\frac{t}{\lambda}}+\frac{f e^{-\gamma B}}{\sqrt{1+\lambda^{2} \omega^{2}}} \cos (\omega t-\gamma A-\psi)+\frac{2 f}{\pi} \int_{0}^{\infty} \frac{\sin (\gamma \xi) e^{-\frac{t}{2 \lambda}}}{\xi\left[\left(\nu \xi^{2}-\lambda \omega^{2}\right)^{2}+\omega^{2}\right]} \\
\times & {\left[\omega^{2} c h\left(\frac{\sqrt{1-4 \lambda \nu \xi^{2}}}{2 \lambda} t\right)-\frac{\omega^{2}+2 \nu \xi^{2}\left(\nu \xi^{2}-\lambda \omega^{2}\right)}{\sqrt{1-4 \lambda \nu \xi^{2}}} \operatorname{sh}\left(\frac{\sqrt{1-4 \lambda \nu \xi^{2}}}{2 \lambda} t\right)\right] d \xi . } \\
\tau_{s M}(y, t) & =\frac{f \lambda \omega}{1+\lambda^{2} \omega^{2}} e^{-\frac{t}{\lambda}}+\frac{f e^{-\gamma B}}{\sqrt{1+\lambda^{2} \omega^{2}}} \sin (\omega t-\gamma A-\psi)+\frac{2 f \omega}{\pi} \int_{0}^{\infty} \frac{\sin (\gamma \xi) e^{-\frac{t}{2 \lambda}}}{\xi\left[\left(\nu \xi^{2}-\lambda \omega^{2}\right)^{2}+\omega^{2}\right]} \\
\times & {\left[\left(\nu \xi^{2}-\lambda \omega^{2}\right) c h\left(\frac{\sqrt{1-4 \lambda \nu \xi^{2}}}{2 \lambda} t\right)+\frac{\nu \xi^{2}+\lambda \omega^{2}}{\sqrt{1-4 \lambda \nu \xi^{2}}} \operatorname{sh}\left(\frac{\sqrt{1-4 \lambda \nu \xi^{2}}}{2 \lambda} t\right)\right] d \xi . }
\end{aligned}
$$

\section{$5.2 \lambda \rightarrow 0$ (second grade fluid)}

Using Equations (27), (28), (42), (43) and the limits

$$
\lim _{\lambda \rightarrow 0} e^{-\frac{b(\xi) t}{2 \lambda}} \operatorname{ch}\left(\frac{c(\xi) t}{2 \lambda}\right)=\lim _{\lambda \rightarrow 0} e^{-\frac{b(\xi) t}{2 \lambda}} \operatorname{sh}\left(\frac{c(\xi) t}{2 \lambda}\right)=\frac{1}{2} \exp \left(\frac{-\gamma \xi^{2} t}{1+\nu \lambda_{r} t}\right),
$$

we obtain solutions corresponding to a fluid of second grade:

$$
u_{c \zeta G}(\gamma, t)=\frac{f}{\mu} \sqrt{\frac{\nu}{\omega}} \frac{e^{-\gamma B}}{\sqrt[4]{1+\delta^{2}}} \cos \left(\omega t-\gamma A+\varphi+\frac{\pi}{2}\right)+\frac{2 f}{\pi \mu\left(1+\delta^{2}\right)} \int_{0}^{\infty} \frac{\xi^{2} \cos (\gamma \xi)}{\left(\xi^{2}+\beta^{2}\right)^{2}+\gamma^{2}} \exp \left(\frac{-\nu \xi^{2} t}{1+\alpha \xi^{2}}\right) d \xi,
$$




$$
\begin{aligned}
& u_{s S G}(y, t)=\frac{f}{\mu} \sqrt{\frac{\nu}{\omega}} \frac{e^{-\gamma B}}{\sqrt[4]{1+\delta^{2}}} \sin \left(\omega t-\gamma A+\varphi+\frac{\pi}{2}\right)-\frac{2 f}{\pi \mu \nu\left(1+\delta^{2}\right)} \int_{0}^{\infty} \frac{\left(1+\alpha \xi^{2}\right) \cos (\gamma \xi)}{\left(\xi^{2}+\beta^{2}\right)^{2}+\gamma^{2}} \exp \left(\frac{-\nu \xi^{2} t}{1+\alpha \xi^{2}}\right) d \xi \\
& \tau_{c S G}(\gamma, t)=f e^{-\gamma B} \cos (\omega t-\gamma A)-\frac{2 f \nu \gamma}{\pi \omega} \int_{0}^{\infty} \frac{\xi^{3} \sin (\gamma \xi)}{\left(\xi^{2}+\beta^{2}\right)^{2}+\gamma^{2} 1+\alpha \xi^{2}} \exp \left(\frac{-\nu \xi^{2} t}{1+\alpha \xi^{2}}\right) d \xi, \\
& \tau_{s S G}(\gamma, t)=f e^{-\gamma B} \sin (\omega t-\gamma A)-\frac{2 f}{\pi} \gamma \int_{0}^{\infty} \frac{\xi^{3} \sin (\gamma \xi)}{\left(\xi^{2}+\beta^{2}\right)^{2}+\gamma^{2}} \exp \left(\frac{-v \xi^{2} t}{1+\alpha \xi^{2}}\right) d \xi,
\end{aligned}
$$

where

$$
\begin{aligned}
\alpha & =v \lambda_{r}, \quad \delta=\frac{\alpha \omega}{v}, \quad \beta^{2}=\frac{\alpha \omega^{2}}{v^{2}\left(1+\delta^{2}\right)}, \quad \gamma=\frac{\omega}{v\left(1+\delta^{2}\right)}, \\
2 A^{2} & =\gamma\left(\sqrt{1+\delta^{2}}-\delta\right), \quad 2 B^{2}=\gamma\left(\sqrt{1+\delta^{2}}+\delta\right), \quad \tan \varphi=\left(\sqrt{1+\delta^{2}}-\delta\right) .
\end{aligned}
$$

\section{$5.3 \lambda \rightarrow 0, \lambda_{r} \rightarrow 0$ (Newtonian fluid)}

Making, $\lambda \rightarrow 0$ and, $\lambda_{r} \rightarrow 0$ into Equations (27), (28), (42), and (43) or, $\lambda \rightarrow 0$ into Equations (44)-(47), or $\alpha=0$ into Equations (48)-(52), we recover the solutions [[23], Equations (20)-(23)]

$$
\begin{aligned}
& u_{c N}(y, t)=\frac{f}{\mu} \sqrt{\frac{\nu}{\omega}} e^{-\gamma \sqrt{\frac{\omega}{2 v}}} \cos \left(\omega t-\gamma \sqrt{\frac{\omega}{2 v}}+\frac{3 \pi}{4}\right)+\frac{2 f}{\mu \pi} \int_{0}^{\infty} \frac{\xi^{2} \cos (\gamma \xi)}{\xi^{4}+\left(\frac{\omega}{v}\right)^{2}} e^{-\nu \xi^{2} t} d \xi, \\
& u_{s N}(\gamma, t)=\frac{f}{\mu} \sqrt{\frac{\nu}{\omega}} e^{-\gamma \sqrt{\frac{\omega}{2 v}}} \sin \left(\omega t-y \sqrt{\frac{\omega}{2 v}}+\frac{3 \pi}{4}\right)-\frac{2 f}{\mu \pi} \frac{\omega}{v} \int_{0}^{\infty} \frac{\cos (\gamma \xi)}{\xi^{4}+\left(\frac{\omega}{v}\right)^{2}} e^{-v \xi^{2} t} d \xi, \\
& \tau_{c N}(y, t)=f e^{-\gamma \sqrt{\frac{\omega}{2 v}}} \cos \left(\omega t-\gamma \sqrt{\frac{\omega}{2 v}}\right)-\frac{2 f}{\pi} \int_{0}^{\infty} \frac{\xi^{3} \sin (\gamma \xi)}{\xi^{4}+\left(\frac{\omega}{v}\right)^{2}} e^{-v \xi^{2} t} d \xi, \\
& \tau_{s N}(y, t)=f e^{-\gamma \sqrt{\frac{\omega}{2 v}}} \sin \left(\omega t-\gamma \sqrt{\frac{\omega}{2 v}}\right)+\frac{2 f}{\pi} \frac{\omega}{v} \int_{0}^{\infty} \frac{\xi \sin (\gamma \xi)}{\xi^{4}+\left(\frac{\omega}{v}\right)^{2}} e^{-\nu \xi^{2} t} d \xi,
\end{aligned}
$$

corresponding to the flow of a Newtonian fluid.

\section{Numerical results and conclusions}

In this article, the unsteady motion of an incompressible Oldroyd-B fluid over an infinite plate that applies an oscillating shear stress to the fluid is studied by means of integral transforms. The starting solutions that have been obtained for velocity and shear stress are presented as a sum of steady-state and transient solutions. They describe the motion of the fluid some time after its initiation. After that time, when the transients disappear, the starting solutions tend to the steady-state solutions that are periodic in time and independent of the initial conditions. However, they satisfy the governing equations and boundary conditions. Furthermore, as it was to be expected, the steady-state solutions corresponding to the cosine oscillations of the shear differ with a phase shift from those due to the sine oscillations of the shear. This 
property is not true for the transient components of solutions. This is the reason that we separately gave the starting solutions for both cosine and sine oscillations of the shear stress on the boundary.

By making $\lambda_{r}=0$ into general solutions (27), (28), (42), and (43), we obtain the similar solutions (44)-(47) corresponding to a Maxwell fluid performing the same motion. Making $\lambda \rightarrow 0$ into Equations (27), (28), (42), and (43) we obtain the similar solutions (48)-(51) corresponding to a second grade fluid performing the same motion. Solutions (44)-(47) with $\lambda \rightarrow 0$, respectively (48)-(51) with $\alpha=0$ give the similar solutions (53)(56) for Newtonian fluids. It is worth pointing out that the expressions of $\tau_{c N}(y, t)$ and $\tau_{s N}(y, t)$ are identical, as form, with those of $v_{c N}(y, t)$ and $v_{s N}(y, t)$ corresponding to a similar motion with the boundary conditions [[13], Equation (3.1)]

$$
v(0, t)=V \cos (\omega t) \quad \text { or } \quad v(0, t)=V \sin (\omega t) ; \quad t>0 .
$$

The velocity field (see [[13], Equations (3.11) and (6.2)])

$$
v_{s N}(\gamma, t)=V e^{-\gamma \sqrt{\frac{\omega}{2 v}}} \sin \left(\omega t-\gamma \sqrt{\frac{\omega}{2 v}}\right)+\frac{2 V}{\pi} \frac{\omega}{v} \int_{0}^{\infty} \frac{\xi \sin (\gamma \xi)}{\xi^{4}+\left(\frac{\omega}{v}\right)^{2}} e^{-v \xi^{2} t} d \xi
$$

for instance, has the same form as $\tau_{s N}(y, t)$ given by Equation (56). This is not a surprise because, for Newtonian fluids, Equations (3) and (4) together with the balance of linear momentum lead to a governing equation for shear stress of the same form as that for velocity.

Generally, the starting solutions for unsteady motions of fluids are important for those who need to eliminate the transients from their rheological measurements. Consequently, an important problem regarding the technical relevance of these solutions is to find the required time to get the steady-state. More exactly, in practice it is necessary to know the approximate time after which the fluid is moving according to the steady-state solutions. For this, the variations of the starting and steady-state velocities with the distance from the plate are depicted in Figures 1, 2, 3, 4, 5, 6, 7, and 8 for sine and cosine oscillations of the shear stress on the boundary. At small values of time, the difference between the starting and steady-state solutions is meaningful. This difference decreases in time and it is clearly seen from figures that the required time to reach the steady-state for the sine oscillations is higher in comparison with the cosine oscillations of the shear. This is obvious, because at $t=0$ the shear stress on the boundary is zero for sine oscillations.

Of course, the required time to reach the steady-state depends on the material constants and the frequency $\omega$ of the shear. Figures 1 and 4 show the influence of $\omega$ on the fluid motion. Furthermore, the required time to reach the steady-state decreases for increasing $\omega$. The influence of the relaxation and retardation times $\lambda$ and $\lambda_{r}$ on the fluid motion is underlined by Figures 2, 3, 5, and 6. The two parameters, as expected, have opposite effects on the motion. The required time to reach the state-state decreases with respect to $\lambda$ and increases with regard to $\lambda_{r}$ for both types of oscillating shear. The steady-state is rather obtained for a Maxwell fluid in comparison with an Oldroyd-B fluid having the same relaxation time $\lambda$. Figures 7 and 8 show the influence of $\omega$ on the motion of second grade and Newtonian fluids. As expected, the required time to reach the steady-state is lower for second grade fluids in comparison with Newtonian fluids. 

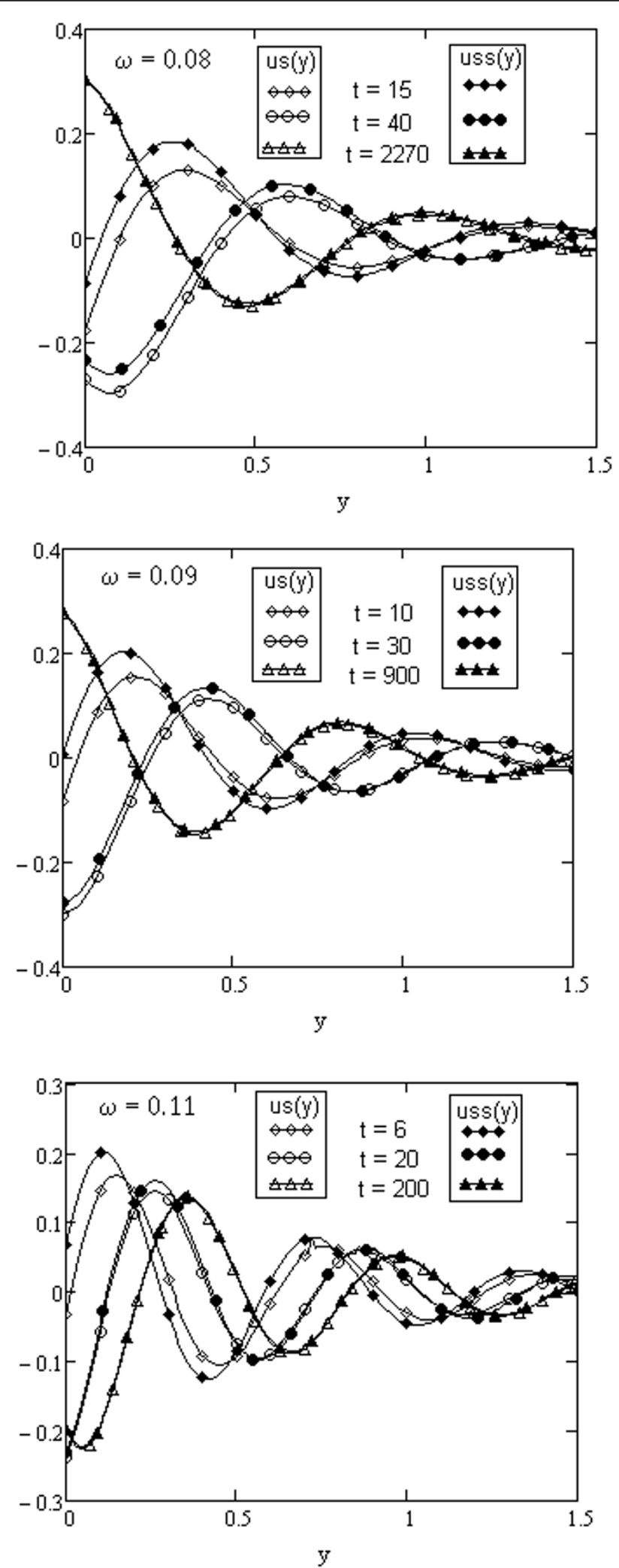

Figure 1 Comparison between the starting velocity $u_{s}(y, t)$ and the steady-state velocity $u_{s s}(y, t)$, for $f=6, v=0.004, \mu=3.92, \lambda=8, \lambda_{r}=4$ and different values of time $t$ and frequency $\omega$. 

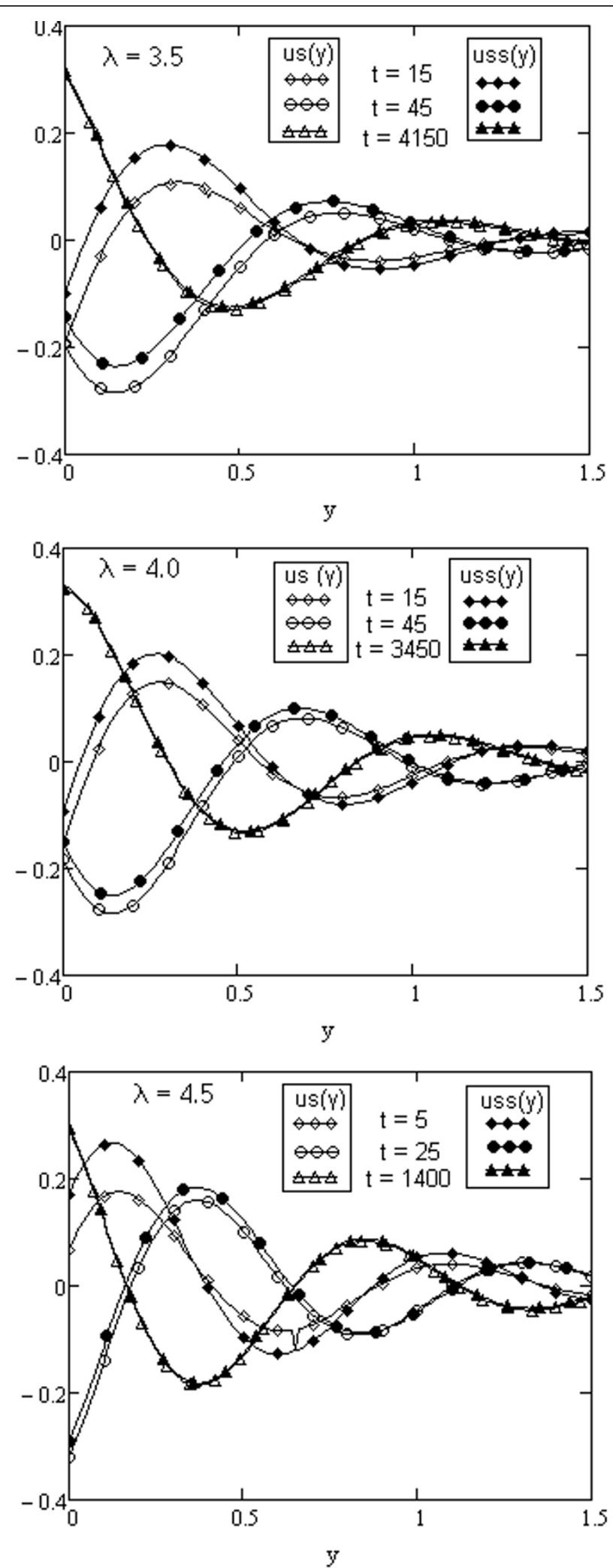

Figure 2 Comparison between the starting velocity $u_{s}(y, t)$ and the steady-state velocity $u_{s s}(y, t)$, for $f=6, v=0.004, \mu=3.92, \lambda_{r}=0.5, \omega=0.08$ and different values of time $t$ and the relaxation time $\lambda$. 

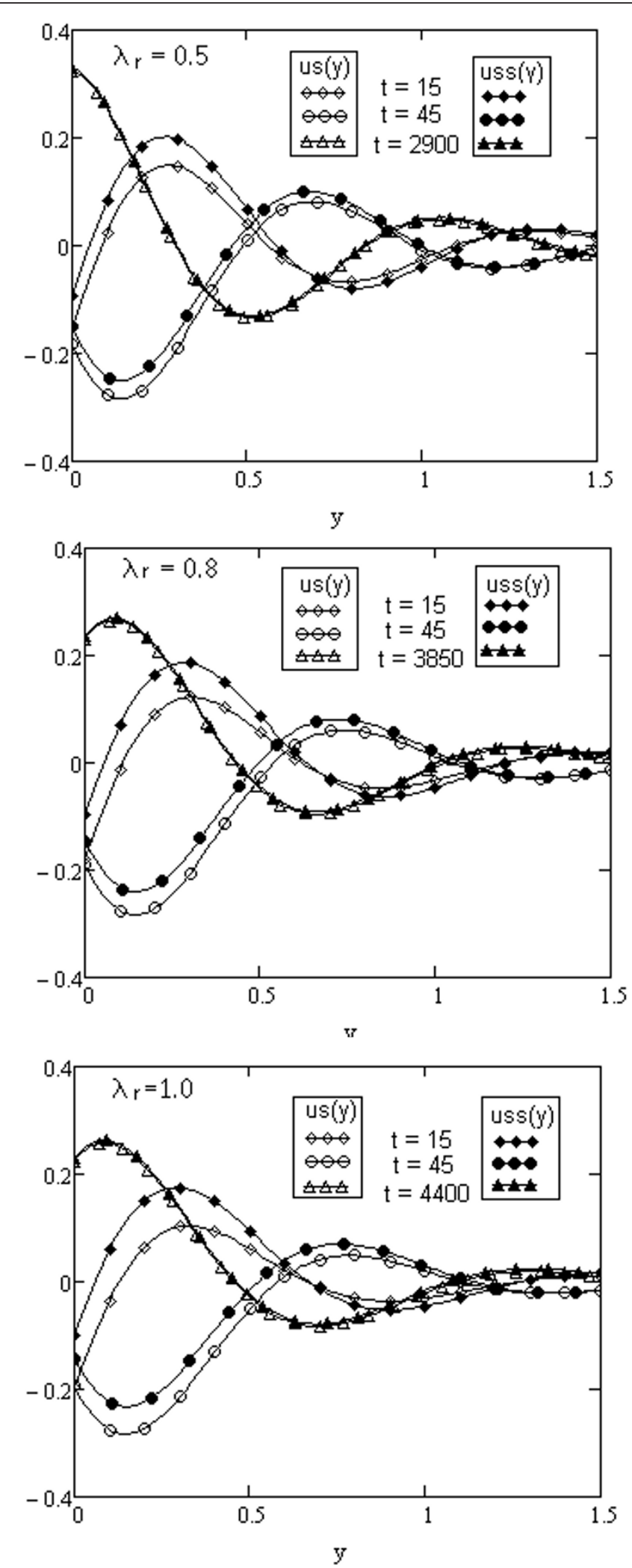

Figure 3 Comparison between the starting velocity $u_{s}(y, t)$ and the steady-state velocity $u_{s s}(y, t)$, for $f=6, v=0.004, \mu=3.92, \lambda=4, \omega=0.08$ and different values of time $t$ and the retardation time $\lambda_{r}$. 

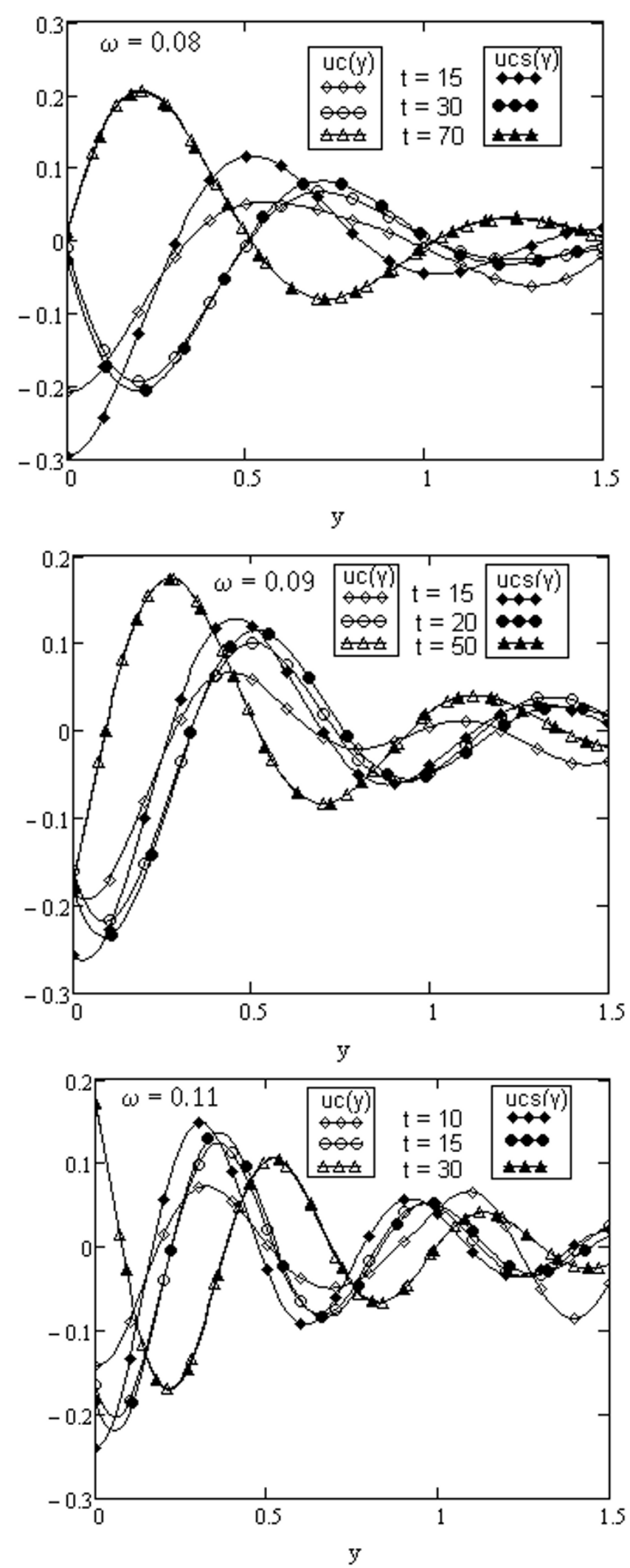

Figure 4 Comparison between the starting velocity $u_{c}(y, t)$ and the steady-state velocity $u_{c s}(y, t)$, for $f=6, v=0.004, \mu=3.92, \lambda=8, \lambda_{r}=4$, and different values of time $t$ and frequency $\omega$. 

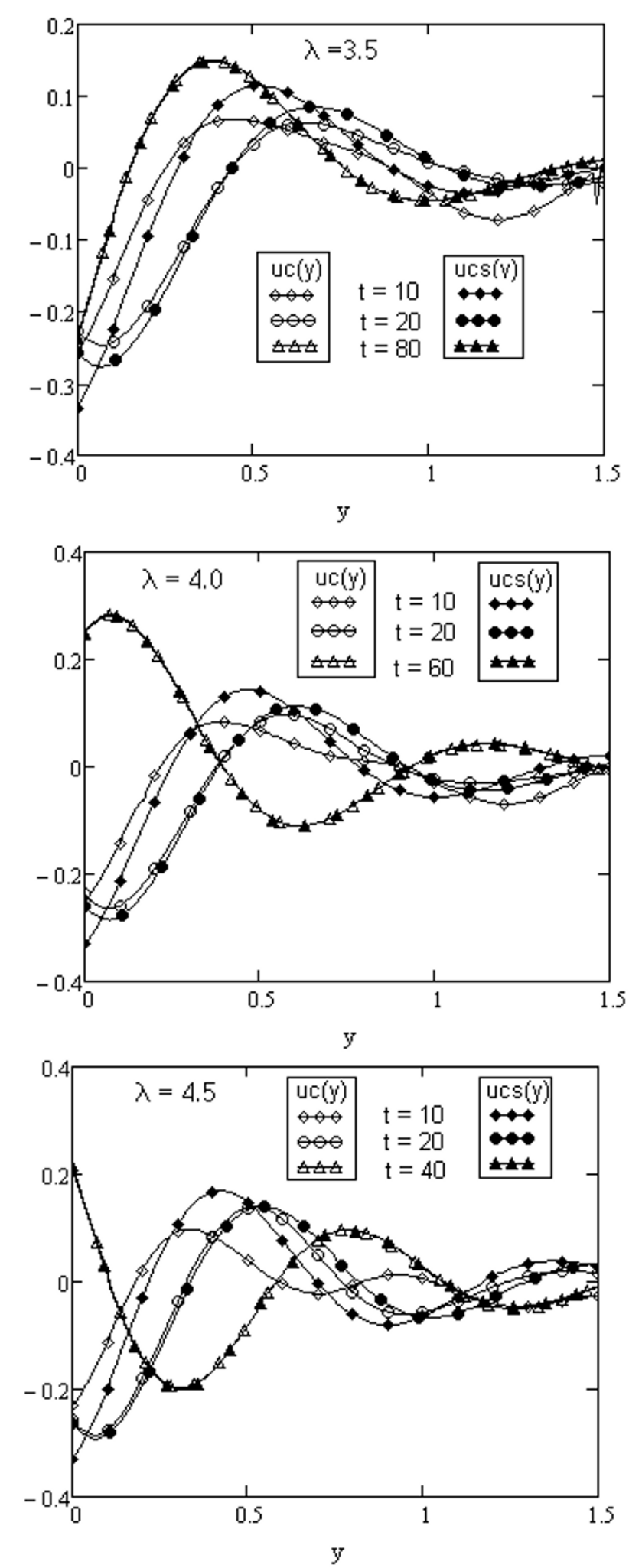

Figure 5 Comparison between the starting velocity $u_{c}(y, t)$ and the steady-state velocity $u_{c s}(y, t)$, for $f=6, v=0.004, \mu=3.92, \lambda_{r}=0.5, \omega=0.08$ and different values of time $t$ and the relaxation time $\lambda$. 

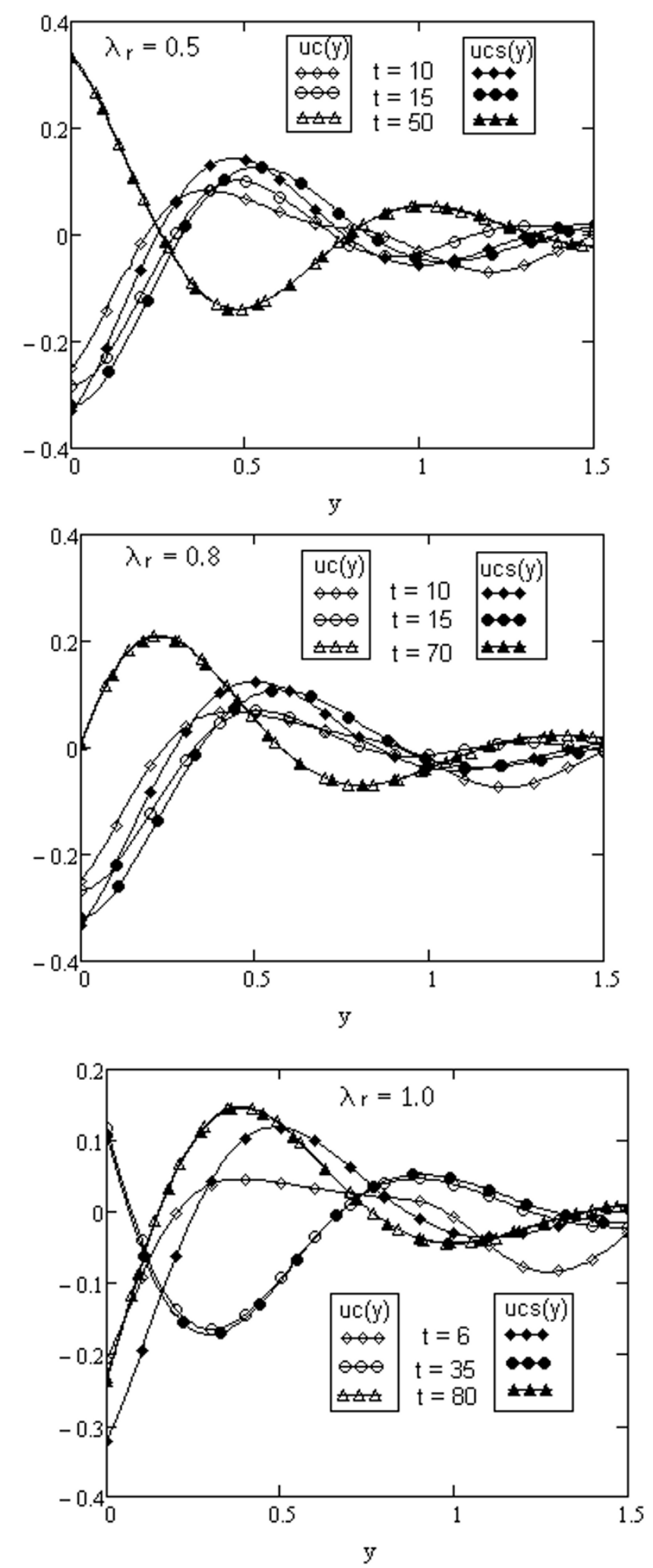

Figure 6 Comparison between the starting velocity $u_{c}(y, t)$ and the steady-state velocity $u_{c s}(y, t)$, for $f=6, v=0.004, \mu=3.92, \lambda=4, \omega=0.08$ and different values of time $t$ and the retardation time $\lambda_{r}$. 


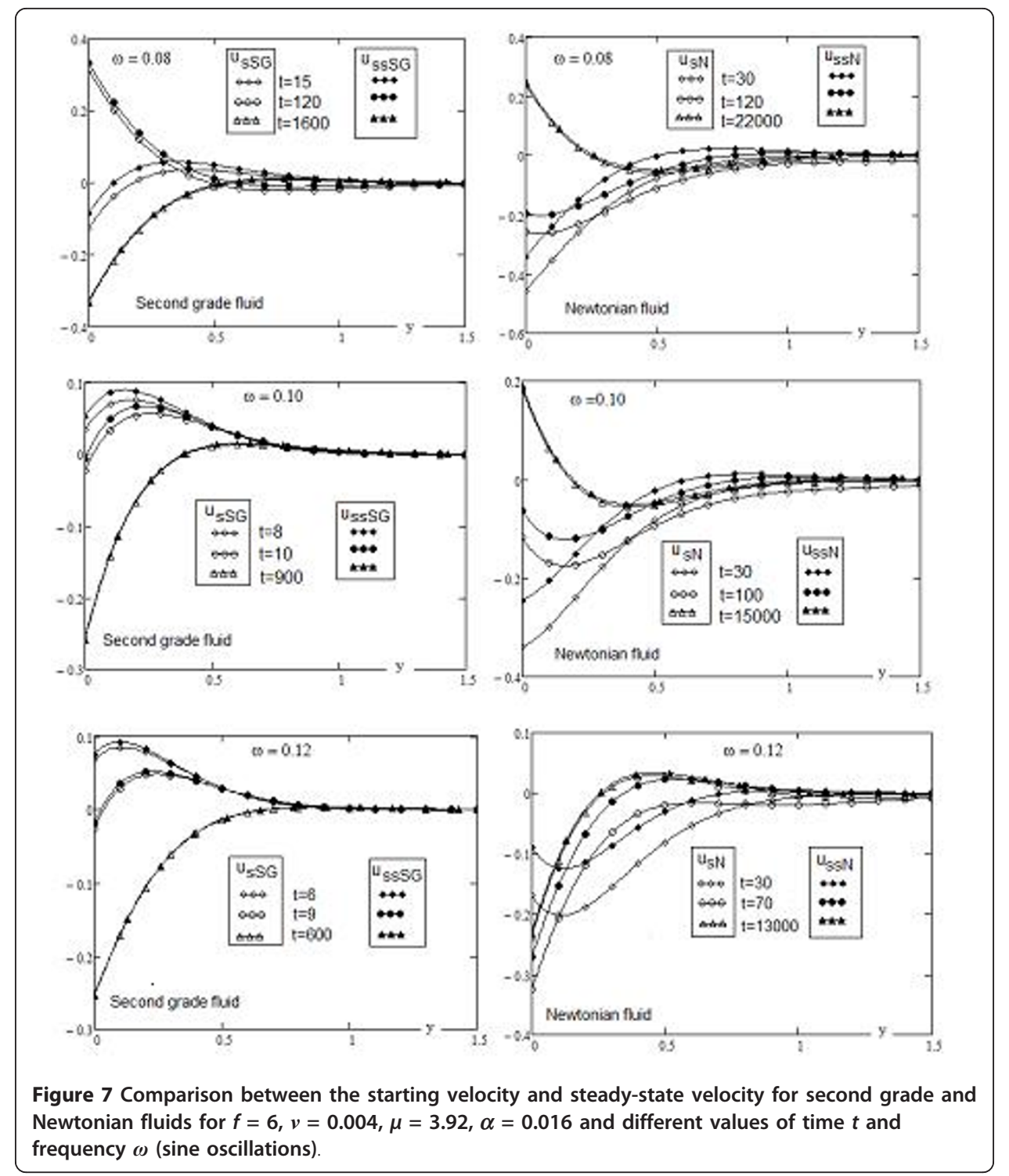

\section{Appendix}

$$
\begin{aligned}
& \int_{0}^{\infty} \frac{x^{2} \cos (m x)}{\left(x^{2}-b^{2}\right)^{2}+c^{2}} d x=\frac{\pi e^{-m B}}{2 c}[A \cos (m A)-B \sin (m A)] \\
& \int_{0}^{\infty} \frac{\cos (m x)}{\left(x^{2}-b^{2}\right)^{2}+c^{2}} d x=\frac{\pi e^{-m B}}{2 c\left(A^{2}+B^{2}\right)}[A \cos (m A)+B \sin (m A)] \\
& \int_{0}^{\infty} \frac{\sin (y \xi)}{\xi}=\frac{\pi}{2} \\
& \int_{0}^{\infty} \frac{x \sin (m x)\left(x^{2}-b^{2}\right)}{\left(x^{2}-b^{2}\right)^{2}+c^{2}} d x=\frac{\pi}{2} e^{-m B} \cos (m A)
\end{aligned}
$$



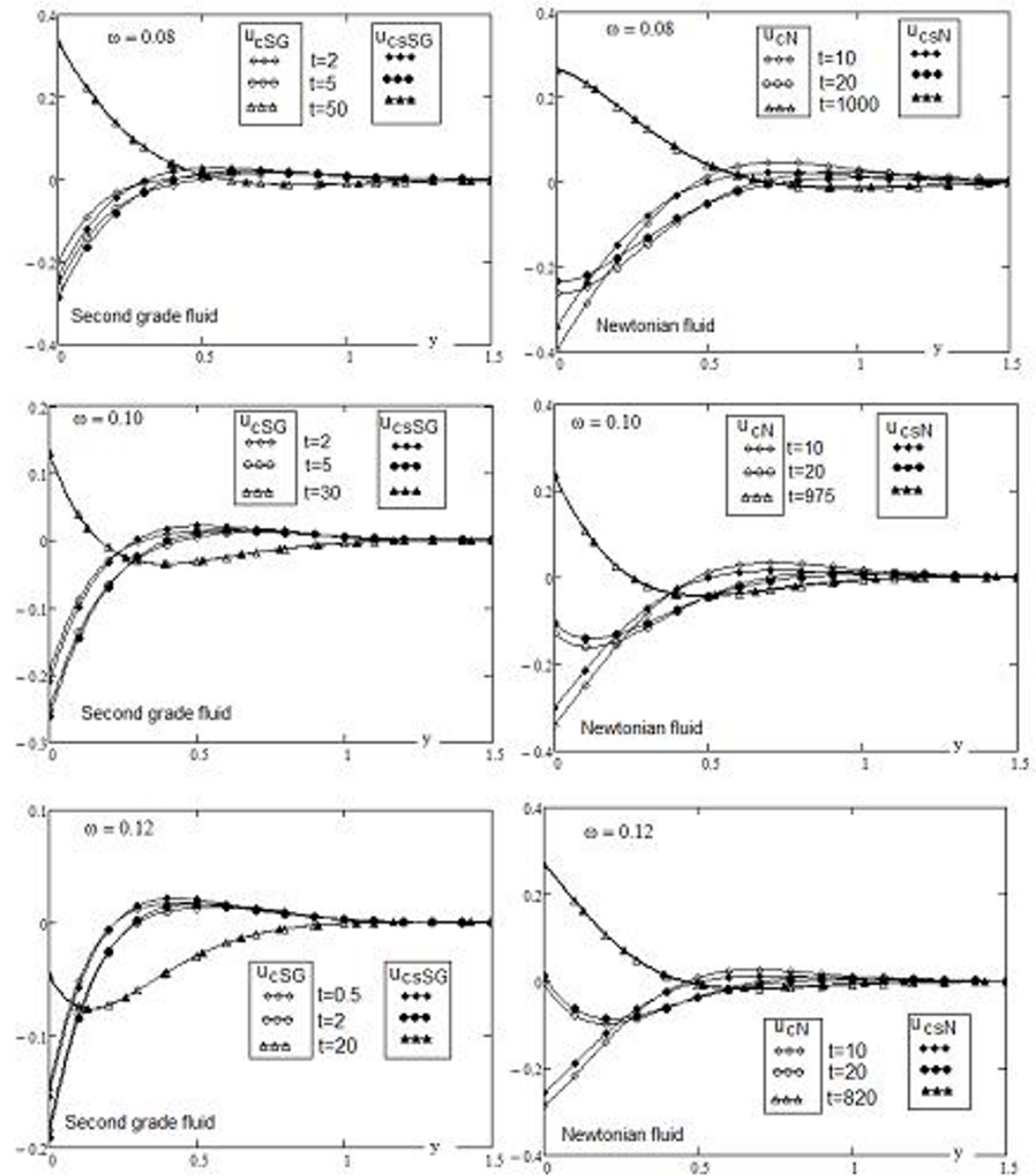

Figure 8 Comparison between the starting velocity and steady-state velocity for second grade and Newtonian fluids for $f=6, v=0.004, \mu=3.92, \alpha=0.016$ and different values of time $t$ and frequency $\omega$ (cosine oscillations)

$$
\begin{aligned}
& \int_{0}^{\infty} \frac{x \sin (m x)}{x\left[\left(x^{2}-b^{2}\right)^{2}+c^{2}\right]} d x=\frac{\pi}{2 c} e^{-m B} \sin (m A), \\
& \int_{0}^{\infty} \frac{\sin (m x)}{x\left[\left(x^{2}-b^{2}\right)^{2}+c^{2}\right]} d x=\frac{\pi}{2 c\left(b^{4}+c^{2}\right)}\left\{c+\left[b^{2} \sin (m A)-c \cos (m A)\right] \cdot \exp (-m B)\right\},
\end{aligned}
$$

where

$$
b^{2}=\frac{\omega^{2}\left(\lambda-\lambda_{r}\right)}{v\left(1+\lambda_{r}^{2} \omega^{2}\right)}, c=\frac{\omega\left(1+\lambda \lambda_{r} \omega^{2}\right)}{v\left(1+\lambda_{r}^{2} \omega^{2}\right)}, 2 A^{2}=\sqrt{b^{4}+c^{2}}+b^{2}, \quad 2 B^{2}=\sqrt{b^{4}+c^{2}}-b^{2} .
$$

The authors would like to express their gratitude to reviewers for fruitful remarks and suggestions regarding the initial version of the manuscript. N. Shahid and M. Rana are highly thankful and grateful to Abdus Salam School of 
Mathematical Sciences GC University, Lahore, Pakistan and Higher Education Commission of Pakistan for generously supporting and facilitating this research work.

\section{Author details}

A Abdus Salam School of Mathematical Sciences, GC University, 68-B New Muslim Town, Lahore, Pakistan ${ }^{2}$ COMSATS Institute of Information Technology, Lahore, Pakistan

\section{Authors' contributions}

The authors' contribution for this article is as follows: NS 35\%, MR 35\%, and IS 30\%. All authors participated in the design of the study and established exact solutions. NS and MR have had a special contribution to get the simplest form of steady-state solutions. IS prepared the most part of graphical illustrations while NS and MR participated in the sequence alignment and drafted the manuscript. All authors participated in drawing conclusions, reading and approving the manuscript.

\section{Competing interests}

The authors declare that they have no competing interests.

Received: 26 November 2011 Accepted: 24 April 2012 Published: 24 April 2012

\section{References}

1. Rajagopal, KR, Srinivasa, AR: A thermodynamic frame work for rate type fluid models. J Non-Newtonian Fluid Mech. 88, 207-227 (2000). doi:10.1016/50377-0257(99)00023-3

2. Tanner, RY: Note of the Rayleigh problem for a visco-elastic fluid. Z Angew Math Phys. 13, 573-580 (1962). doi:10.1007/ BF01595580

3. Waters, ND, King, MJ: Unsteady flow of an elastico-viscous liquid. Rheol Acta. 9, 345-355 (1970). doi:10.1007/BF01975401

4. Rajagopal, KR: A note on unsteady unidirectional flows of a non-Newtonian fluid. Int J Non-linear Mech. 17, 369-373 (1982). doi:10.1016/0020-7462(82)90006-3

5. Erdogan, ME: A note on an unsteady flow of a viscous fluid due to an oscillating plane wall. Int J Non-linear Mech. 35 1-6 (2000). doi:10.1016/S0020-7462(99)00019-0

6. Penton, R: The transient for Stokes' oscillating plane: a solution in terms of tabulated functions. J Fluid Mech. 31 , 819-825 (1968). doi:10.1017/S0022112068000509

7. Rajagopal, KR: Longitudinal and torsional oscillations of a rod in a non-Newtonian fluid. Acta Mech. 49, $281-285$ (1983). doi:10.1007/BF01236358

8. Puri, P, Kythe, PK: Stokes' first and second problems for Rivlin-Ericksen fluids with nonclassical heat conduction. ASME J Heat Trans. 120, 44-50 (1998), doi:10.1115/1.2830061

9. Rajagopal, KR, Bhatnagar, RK: Exact solutions for some simple flows of an Oldroyd-B fluid. Acta Mech. 113, 233-239 (1995). doi:10.1007/BF01212645

10. Hayat, T, Asghar, S, Siddiqui, AM: Periodic unsteady flows of a non-Newtonian fluid. Acta Mech. 131, $169-175$ (1998). doi:10.1007/BF01177223

11. Siddiqui, AM, Hayat, T, Asghar, S: Periodic flows of a non-Newtonian fluid between two parallel plates. Int J Non-linear Mech. 34, 895-899 (1999). doi:10.1016/S0020-7462(98)00063-8

12. Fetecau, C, Fetecau, C: Starting solutions for some unsteady unidirectional flows of a second grade fluid. Int J Eng Sci. 43, 781-789 (2005). doi:10.1016/j.ijengsci.2004.12.009

13. Fetecau, C, Fetecau, C: Starting solutions for the motion of a second grade fluid due to longitudinal and torsional oscillations of a circular cylinder. Int J Eng Sci. 44, 788-796 (2006). doi:10.1016/j.ijengsci.2006.04.010

14. Aksel, N, Fetecau, C, Scholle, M: Starting solutions for some unsteady unidirectional flows of Oldroyd-B fluids. Z Angew Math Phys. 57, 815-831 (2006). doi:10.1007/s00033-006-0063-8

15. Vieru, D, Akhtar, W, Fetecau, C, Fetecau, C: Starting solutions for the oscillating motion of a Maxwell fluid in cylindrical domains. Mecca. 42, 573-583 (2007). doi:10.1007/s11012-007-9081-7

16. Nazar, M, Fetecau, C, Vieru, D, Fetecau, C: New exact solutions corresponding to the second problem of Stokes for second grade fluids. Nonlinear Anal Real World Appl. 11, 584-591 (2008)

17. Fetecau, C, Hayat, T, Fetecau, C: Starting solutions for oscillating motions of Oldryd-B fluids in cylindrical domains. Int J Non-Newtonian Fluid Mech. 153, 191-201 (2008). doi:10.1016/j.jnnfm.2008.02.005

18. Zheng, L, Zhao, F, Zhang, X: Exact solutions for generalized Maxwell fluid flow due to oscillatory and constantly accelerating plate. Nonlinear Anal Real World Appl. 11, 3744-3751 (2010). doi:10.1016/j.nonrwa.2010.02.004

19. Anjum, A, Ayub, M, Khan, M: Starting solutions for oscillating motions of an Oldroyd-B fluid over a plane wall. Commun Nonlinear Sci Numer Simul. 17, 472-482 (2012). doi:10.1016/j.cnsns.2011.05.004

20. Khan, M, Arshad, M, Anjum, A: On exact solutions of Stokes second problem for MHD Oldroyd-B fluid. Nucl Eng Des. 243, 20-32 (2012)

21. Waters, ND, King, MJ: Unsteady flow of an elastico-viscous liquid. Rheol Acta. 9, 345-355 (1970). doi:10.1007/BF01975401

22. Bandelli, R, Rajagopal, KR, Galdi, GP: On some unsteady motions of fluids of second grade. Arch Mech (Arch Mech Stos). 47, 661-676 (1995)

23. Fetecau, C, Vieru, D, Fetecau, C: E ${ }^{\oplus}$ ect of the side walls on the motion of a viscous fluid induced by an infinite plate that applies an oscillating shear stress to the fluid. Cent Eur J Phys. 9, 816-825 (2011). doi:10.2478/s11534-010-0073-1

24. Fetecau, C, Prasad, SC, Rajagopal, KR: A note on the flow induced by a constantly accelerating plate in an Oldryod-B fluid. Appl Math Model. 31, 647-654 (2007). doi:10.1016/j.apm.2005.11.032

25. Integral Transforms and Their Applications. Chapman and Hall, Boca-Raton (2007)

26. Sneddon, IN: Fourier Transforms. McGraw Hill, New York (1951) 